\title{
Complete Electromagnetic Dyadic Green Function Characterization in a Complex Environment-Resonant Dipole-Dipole Interaction and Cooperative Effects
}

\author{
Kaizad Rustomji, ${ }^{1,2}$ Marc Dubois, ${ }^{1}$ Pierre Jomin, ${ }^{1}$ Stefan Enoch $\odot,{ }^{1}$ Jérôme Wenger®, ${ }^{1, *}$ \\ C. Martijn de Sterke, ${ }^{2, \dagger}$ and Redha Abdeddaim $\circledast^{1, \$}$ \\ ${ }^{1}$ Aix Marseille Univ, CNRS, Centrale Marseille, Institut Fresnel, Marseille 13013, France \\ ${ }^{2}$ Institute for Photonics and Optical Sciences (IPOS), School of Physics, University of Sydney, \\ Sydney, New South Wales 2006, Australia
}

(Received 26 March 2020; revised 7 December 2020; accepted 4 February 2021; published 5 April 2021)

\begin{abstract}
The Green function completely encapsulates a system's linear response to external sources, and plays a central role in optics, electromagnetism, and acoustics. In electromagnetism, a broad range of phenomena are connected to the Green function, including the local density of optical states, superradiance, and the cooperative Lamb shift. Therefore, knowing the Green function is important for progress in fields as diverse as cavity quantum electrodynamics, plasmonics, metamaterials, and photovoltaics. However, experimentally characterizing the full complex Green function is challenging, as it requires amplitude and phase sensitive measurements with deep-subwavelength spatial resolution. Here, we report a method to characterize the full complex Green function with a resolution of $\lambda / 100$ by measuring the mutual impedance between two dipoles at microwave frequencies. We apply it to a resonant planar cavity, with both parallel and nonparallel sides, and also explore the effects of modal resonances in a dielectric cube on dipole-dipole interactions. The ability to characterize the Green function with high spatial resolution provides a unique way to investigate cooperative effects in complex photonic systems.
\end{abstract}

DOI: 10.1103/PhysRevX.11.021004

\section{INTRODUCTION}

The dyadic Green function is a central element in the theory describing many areas of physics as it encapsulates the linear response of a complex environment to an arbitrary distribution of sources. In classical electrodynamics, the Green function $\stackrel{\leftrightarrow}{\boldsymbol{G}}\left(\boldsymbol{r}_{A}, \boldsymbol{r}_{D} ; \omega\right)$ corresponds to the electric field at a position $\boldsymbol{r}_{A}$ emitted from a point source dipole at position $\boldsymbol{r}_{D}$ [1]. In general, it is a frequencydependent complex tensor function of both the source and observer positions. The Green function encompasses all the information to calculate the system's response, and as such its tensor elements account for a broad range of physical phenomena For instance, the imaginary part of the diagonal elements allows computation of the (partial) local density of optical states (LDOS) and the associated Purcell factor $[2,3]$, which determine the efficiency of a classical antenna [4] and the lifetime of a quantum emitter in a complex

\footnotetext{
*jerome.wenger@fresnel.fr †martijn.desterke@sydney.edu.au redha.abdeddaim@fresnel.fr
}

Published by the American Physical Society under the terms of the Creative Commons Attribution 4.0 International license. Further distribution of this work must maintain attribution to the author(s) and the published article's title, journal citation, and DOI.
Subject Areas: Nanophysics, Photonics

environment [5]. The imaginary part of the off-diagonal elements of the Green function determines the cross density of optical states (CDOS) to characterize the spatial coherence in complex systems [6,7]. Different aspects of the resonant dipole-dipole interaction (RDDI), an example of the collective effects possible between several elementary dipoles, are also determined by the Green function: the cooperative decay rate (CDR) [8-10] is determined by the imaginary parts of the tensor elements, the cooperative Lamb shift (CLS) $[10,11]$ is determined by the real parts of the tensor elements, and the Förster resonance energy transfer (FRET) is determined by the square moduli of the tensor elements [12-14]. The Green function is thus a powerful paradigm in many areas of wave physics, including cavity quantum electrodynamics (cavity QED) [15-22], plasmonics and metamaterials [23-27], wave front shaping and time-reversal focusing [28,29], or acoustics [30-32]. In addition to this, the Green function is central in solving inverse problems in which the distribution of sources needs to be determined from the knowledge of the field [33].

Although the Green function is a central concept, it is only fully known analytically for relatively simple geometries, such as free space [1], infinite isotropic and anisotropic media [34], multilayer stacks [35], photonic crystals [36], and in the presence of infinite, ideally conducting plates [37]. Experimental measurements of the Green function could provide in-depth information 
about a wider range of systems and configurations, yet a major challenge is that the Green function needs to be known in both amplitude and phase at a high spatial resolution, well below the wavelength. These highly demanding requirements have significantly limited experimental attempts to measure the Green function. In optics, experiments are challenging due to the weak signals involved and the low spatial resolution [38-40], while in electron energy loss spectroscopy, highly specialized equipment and inversion approaches are needed [41-43]. In contrast, experiments performed in the microwave regime benefit from direct measurements of the complex electric field, small detection bandwidths, and spatial resolutions well below the wavelength [44-47]. However, no complete amplitude and phase characterization of the Green function has been reported so far.

Here, we report a method to experimentally determine the full complex Green function at spatial resolutions as low as $\lambda / 100$, by measuring the mutual impedance between two dipoles at microwave frequencies. To demonstrate the effectiveness of our approach, we fully characterize the Green function inside a resonant planar cavity of parallel or nonparallel mirrors. This information quantifies the influence of the cavity on various aspects of resonant dipoledipole interaction (CDR, CLS, and FRET), and is in excellent agreement with classical electrodynamics simulations. We also explore how the energy transfer between two dipoles is mediated by a resonant dielectric subwavelength cube in different configurations.

Our novel methodology allows the full experimental characterization of the Green function in both amplitude and phase at ultrahigh spatial resolution. This provides a powerful way to solve problems for which no analytic solution exists and where numerical simulations demand excessive computational resources. Although the measurements are performed at microwave frequencies, the results of the Green function characterization can be scaled to provide relevant information in the visible regime, where no such measurements are feasible. This allows the optimization of the design of photonic structures to enhance resonant dipole-dipole interactions and cooperative effects. Even though at microwave frequencies metals have very low loss compared to the visible regime, it is possible to mimic losses, finite conductivities, and surface plasmons at microwave frequencies by using structured surfaces and volumes [47-49]. This further broadens the applicability of our approach to the visible range.

By providing a unified quantitative description of RDDI inside a cavity, our results are highly relevant for cavity QED [15-22] and cavity-enhanced light-matter interactions [37,50-53]. The experimental characterization of the Green function is also crucial for solving problems related to open cavities or for configurations for which no analytic solution exists and for which numerical approaches are very extensive or even impractical. Altogether, the general methodology described here can be broadly applied to characterize the electromagnetic response of a wide range of systems at ultrahigh spatial resolution and to improve our understanding of the rich physics of dipole-dipole interactions.

\section{THEORY}

We start by briefly reviewing how the Green function relates to the different aspects of dipole radiation and resonant dipole-dipole interaction. The electric field $\boldsymbol{E}_{D}\left(\boldsymbol{r}_{A}\right)$ generated from a point source dipole at position $\boldsymbol{r}_{D}$ and evaluated at a position $\boldsymbol{r}_{A}$ defines the Green function $\stackrel{\leftrightarrow}{\boldsymbol{G}}\left(\boldsymbol{r}_{A}, \boldsymbol{r}_{D}\right)$ as [1]

$$
\boldsymbol{E}_{D}\left(\boldsymbol{r}_{A}\right)=\frac{\omega^{2}\left|\mu_{D}\right|}{c^{2} \varepsilon_{0}} \stackrel{\leftrightarrow}{\boldsymbol{G}}\left(\boldsymbol{r}_{A}, \boldsymbol{r}_{D}\right) \boldsymbol{n}_{D},
$$

where $\left|\mu_{D}\right| \boldsymbol{n}_{D}$ is the donor's dipole moment. The (partial) local density of optical states determining the emission from a single dipole is then given by [2,3] (partial means that it is projected on the dipole axis which we assume to be fixed)

$$
\rho_{\mathrm{LDOS}}=2 \frac{\omega}{\pi c^{2}} \boldsymbol{n}_{D} \cdot \operatorname{Im}\left\{\stackrel{\leftrightarrow}{\boldsymbol{G}}\left(\boldsymbol{r}_{D}, \boldsymbol{r}_{D}\right)\right\} \cdot \boldsymbol{n}_{D}
$$

With this definition, the spontaneous decay rate is given by $\gamma=\left(\pi \omega / \hbar \varepsilon_{0}\right)\left|\mu_{D}\right|^{2} \rho_{\text {LDOS }}$ [1]. The LDOS concept can be extended to two emitters located at $\boldsymbol{r}_{D}$ and $\boldsymbol{r}_{A}$ to characterize the spatial coherence by the cross density of optical states $[6,7]$ :

$$
\rho_{\mathrm{CDOS}}=2 \frac{\omega}{\pi c^{2}} \boldsymbol{n}_{A} \cdot \operatorname{Im}\left\{\stackrel{\leftrightarrow}{\boldsymbol{G}}\left(\boldsymbol{r}_{A}, \boldsymbol{r}_{D}\right)\right\} \cdot \boldsymbol{n}_{D}
$$

By comparing Eqs. (2) and (3), it is apparent that the LDOS is the limit of the CDOS when $\boldsymbol{r}_{A}$ approaches $\boldsymbol{r}_{D}$. Both the CDOS and the LDOS depend on the imaginary part of the Green function, which is shown in Fig. 1(a) for two dipoles in vacuum [1]. For short dipole-dipole distances in the near field such that $R \equiv\left|\boldsymbol{r}_{A}-\boldsymbol{r}_{D}\right|<\lambda$, the CDOS and the imaginary part of the Green function are nearly constant and remain finite when $R \rightarrow 0$. Oscillations of the Green function occur for larger distances $k R>3$ in the far-field region, where the electric field propagates radiatively.

The Green function also determines the cooperative effects in the resonant dipole-dipole interaction. For two dipoles of moments $\left|\mu_{A}\right| \boldsymbol{n}_{A}$ and $\left|\mu_{D}\right| \boldsymbol{n}_{D}$, the cooperative decay rate describing superradiance is given by [8-10] 


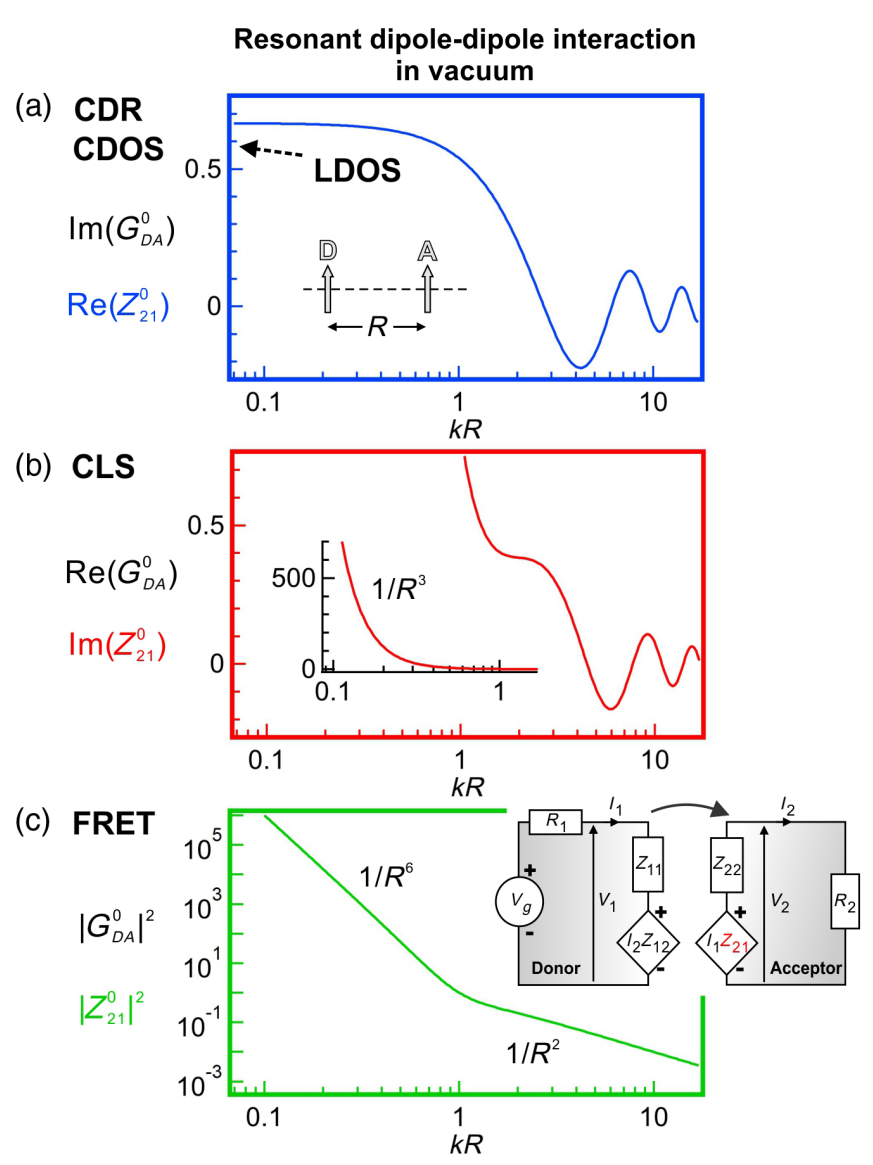

FIG. 1. Complex-valued Green function in vacuum describing the RDDI versus the dipole-dipole separation $R$. (a) Imaginary part of the Green function, which is related to the cooperative decay rate (CDR) and cross density of states (CDOS). It converges to the LDOS as $R \rightarrow 0$. The inset describes the dipoles' mutual orientations considered here. (b) Real part of the Green function, which determines the cooperative Lamb shift (CLS). In contrast to the imaginary part, the real part of the Green function diverges as $R \rightarrow 0$. (c) Square modulus of the Green function, which is linked to the energy transfer FRET. $G_{D A}^{0}$ is the projection of the Green tensor on the dipole direction in homogeneous space which is related to the mutual impedance $Z_{21}^{0}$ between the two dipoles. The inset, showing the two-port network model of dipole-dipole interaction, illustrates how the mutual impedance may be measured.

$$
\gamma_{\mathrm{CDR}}=2 \frac{\omega^{2}\left|\mu_{A}\right|\left|\mu_{D}\right|}{\hbar \varepsilon_{0} c^{2}} \boldsymbol{n}_{A} \cdot \operatorname{Im}\left\{\stackrel{\leftrightarrow}{\boldsymbol{G}}\left(\boldsymbol{r}_{A}, \boldsymbol{r}_{D}\right)\right\} \cdot \boldsymbol{n}_{D}
$$

Thus, we find the same dependence on the imaginary part of the Green function for CDR and for CDOS, as both quantities are related by $\gamma_{\mathrm{CDR}}=\left(\pi \omega / \hbar \varepsilon_{0}\right)\left|\mu_{A}\right|\left|\mu_{D}\right| \rho_{\mathrm{CDOS}}$. Therefore, the CDR and the CDOS have the same relationship to the Green function.

The cooperative Lamb shift, a frequency shift of a collection of emitters, is obtained from [10,11]

$$
J_{\mathrm{CLS}}=-\frac{\omega^{2}\left|\mu_{A}\right|\left|\mu_{D}\right|}{\hbar \varepsilon_{0} c^{2}} \boldsymbol{n}_{A} \cdot \operatorname{Re}\left\{\stackrel{\leftrightarrow}{\boldsymbol{G}}\left(\boldsymbol{r}_{A}, \boldsymbol{r}_{D}\right)\right\} \cdot \boldsymbol{n}_{D}
$$

Thus, the CLS depends on the real part of the Green function. In contrast to the imaginary part, the real part of $\stackrel{\leftrightarrow}{\boldsymbol{G}}\left(\boldsymbol{r}_{A}, \boldsymbol{r}_{D}\right)$ diverges as the dipole-dipole distance $R \rightarrow 0$ [Fig. 1(b)]. Owing to its $1 / R^{3}$ distance dependence, the real part of $\stackrel{\leftrightarrow}{\boldsymbol{G}}\left(\boldsymbol{r}_{A}, \boldsymbol{r}_{D}\right)$ quickly dominates over its imaginary counterpart in the near-field region where $k R<1$, where $k=2 \pi / \lambda$ is the free-space wave number [1].

The final phenomenon related to dipole-dipole interaction is the Förster resonance energy transfer describing the power transferred from a donor to an acceptor $[1,44]$ :

$$
\gamma_{\mathrm{FRET}}=\frac{\omega^{5}\left|\mu_{D}\right|^{2}}{2 c^{4} \varepsilon_{0}^{2}} \operatorname{Im}\left\{\alpha_{A}\right\}\left|\boldsymbol{n}_{A} \cdot \stackrel{\leftrightarrow}{\boldsymbol{G}}\left(\boldsymbol{r}_{A}, \boldsymbol{r}_{D}\right) \boldsymbol{n}_{D}\right|^{2},
$$

where $\alpha_{A}$ is the acceptor dipole's polarizability. Equation (6) shows that FRET depends on the square modulus of the Green function, thus involving both the real and imaginary parts. Figure 1(c) illustrates the expected distance behavior for two dipoles in vacuum, with the FRET power scaling as $1 / R^{6}$ (evanescent coupling) in the near-field region and $1 / R^{2}$ (radiative coupling) in the farfield region [1].

We now describe how to determine the Green function by measuring the mutual impedance between the two dipoles in the microwave regime. A two-port network model describes the resonant dipole-dipole interaction at microwave frequencies [Fig. 1(c)] [54]. Port 1 represents the donor dipole, with voltage $V_{1}$ and current $I_{1}$ driven by a source voltage $V_{g}$. Port 2 corresponds to the acceptor dipole with voltage $V_{2}$ and current $I_{2}$. This port has no driving source, but is coupled to the voltage and current from port 1 through the $Z$ matrix [54]:

$$
\left(\begin{array}{l}
V_{1} \\
V_{2}
\end{array}\right)=\left(\begin{array}{ll}
Z_{11} & Z_{12} \\
Z_{21} & Z_{22}
\end{array}\right)\left(\begin{array}{l}
I_{1} \\
I_{2}
\end{array}\right) .
$$

In the absence of current $I_{2}$, the mutual impedance $Z_{21}$ is defined by the ratio of the induced voltage $V_{2}$ by the source current $I_{1}$ [54]:

$$
Z_{21}=\left.\frac{V_{2}}{I_{1}}\right|_{I_{2}=0} .
$$

The voltage $V_{2}$ depends on the donor's electric field $\boldsymbol{E}_{D}\left(\boldsymbol{r}_{A}\right)$ generated in the acceptor circuit. Using the dipole approximation, which is justified since the dipole lengths are much shorter than the wavelength, we may consider $\boldsymbol{E}_{D}\left(\boldsymbol{r}_{A}\right)$ to be uniform over the acceptor antenna length $l_{A}$. Therefore, we can write $V_{2}=\boldsymbol{n}_{A} \cdot \boldsymbol{E}_{D}\left(\boldsymbol{r}_{A}\right) l_{A}$ [54]. Following Eq. (1), which serves to define the Green function, the voltage $V_{2}$, the electric field $\boldsymbol{E}_{D}\left(\boldsymbol{r}_{A}\right)$, and the Green 
function $\stackrel{\leftrightarrow}{\boldsymbol{G}}\left(\boldsymbol{r}_{A}, \boldsymbol{r}_{D}\right)$ are all directly proportional to each other.

For antenna lengths that are short compared to the wavelength, the source current is given by $I_{1}=-i \omega \mu_{D} / l_{D}$, where $l_{D}$ is the donor's dipole length $[54,55]$. The definition Eq. (8) of the mutual impedance $Z_{21}$ can thus be rewritten as

$$
Z_{21}=i \frac{\omega l_{A} l_{D}}{c^{2} \varepsilon_{0}} \boldsymbol{n}_{A} \cdot \stackrel{\leftrightarrow}{\boldsymbol{G}}\left(\boldsymbol{r}_{A}, \boldsymbol{r}_{D}\right) \cdot \boldsymbol{n}_{D}
$$

Thus, $Z_{21}$ is directly proportional to the projected Green function $\stackrel{\leftrightarrow}{\boldsymbol{G}}\left(\boldsymbol{r}_{A}, \boldsymbol{r}_{D}\right)$. Measuring the full, complex $Z_{21}$ using a microwave network analyzer then provides a direct way to characterize the Green function in an arbitrary environment. The real part of $Z_{21}$ is related to the imaginary part of $\stackrel{\leftrightarrow}{\boldsymbol{G}}\left(\boldsymbol{r}_{A}, \boldsymbol{r}_{D}\right)$ and thus to the CDR and the CDOS:

$$
\gamma_{\mathrm{CDR}}=2 \frac{\omega\left|\mu_{A}\right|\left|\mu_{D}\right|}{\hbar l_{A} l_{D}} \operatorname{Re}\left\{Z_{21}\right\} .
$$

The imaginary part of $Z_{21}$ provides the real part of $\stackrel{\leftrightarrow}{\boldsymbol{G}}\left(\boldsymbol{r}_{A}, \boldsymbol{r}_{D}\right)$ determining the CLS:

$$
J_{\mathrm{CLS}}=-\frac{\omega\left|\mu_{A}\right|\left|\mu_{D}\right|}{\hbar l_{A} l_{D}} \operatorname{Im}\left\{Z_{21}\right\},
$$

while the square modulus of $Z_{21}$ is related to the power transferred by FRET:

$$
\gamma_{\mathrm{FRET}}=\frac{\omega^{3}\left|\mu_{D}\right|^{2}}{2 l_{A}^{2} l_{D}^{2}} \operatorname{Im}\left\{\alpha_{A}\right\}\left|Z_{21}\right|^{2} .
$$

The set of equations (10)-(12) unifies the different descriptions of RDDI using CDOS, CDR, CLS, or FRET. By connecting the mutual impedance $Z_{21}$ to CDR, CLS, and FRET, it further extends the analogies between microwave engineering and quantum and semiclassical electrodynamics [56,57]. By measuring the complex impedance at microwave frequencies, both amplitude and phase information about the Green function can be obtained with excellent subwavelength spatial resolution. As the laws of electromagnetism are scalable with the frequency (provided the material permittivities are similar), the characterization of resonant dipole-dipole interaction near structures in the microwave regime can be readily extended to also explain the physics occurring in the visible range. Microwave experiments can be used as guidelines for near-infrared and visible regime, where no such fully resolved measurements of the Green function are possible.

\section{RESULTS AND DISCUSSION}

We apply our methodology to characterize how a planar cavity modifies dipole-dipole cooperative effects. Planar resonant cavities are frequently used in cavity QED [15-21] and cavity-enhanced light-matter interactions [37,50-53]. Figure 2(a) shows our configuration. Two dipoles (a donor and an acceptor) are set parallel to each other and to the mirrors forming the planar cavity, which can be considered to be infinite in the transverse directions. For this configuration, there is a cutoff for cavity lengths $L<\lambda / 2$ for which no propagating mode exists, and resonances are expected when $L$ equals multiples of $\lambda / 2$. For the experiments, the source donor dipole and the acceptor dipole are connected to a vector network analyzer (VNA) to serve as the two ports of the model in Fig. 1(c). The VNA records the amplitude and the phase of the $Z$ matrix elements, allowing retrieval of the variations of the real and imaginary parts of the Green function $\stackrel{\leftrightarrow}{\boldsymbol{G}}\left(\boldsymbol{r}_{A}, \boldsymbol{r}_{D}\right)$ and comparison to reference values in the absence of the cavity.

Following the approach in Ref. [46], the Purcell factor defining the LDOS modifications is determined by the ratio of the real parts of the $Z$ matrix element $Z_{11}$ in the presence and absence of the cavity. The experimental results are presented in Fig. 2(b) (thin colored curves). Note the excellent agreement with computations derived from the Green function (thick black lines), which is known in analytic form for this case and has no adjustable parameters $[37,58,59]$. In particular, the cutoff for lengths below $\lambda / 2$, the resonances for cavity lengths that are multiples of $\lambda / 2$, and the suppression just below these resonances clearly show in our experimental results.

We next investigate the RDDI inside the cavity by measuring the real and imaginary parts of the mutual impedance $Z_{21}$ demonstrating that we can fully characterize the Green function projected onto the dipole axes. Figures 2(c)-2(k) quantify the evolutions of the real and imaginary parts of $\stackrel{\leftrightarrow}{\boldsymbol{G}}\left(\boldsymbol{r}_{A}, \boldsymbol{r}_{D}\right)$ determining the different physical phenomena (CDR, CDOS, CLS, FRET). All the experimental data in Figs. 2(c)-(k) and Figs. S1 and S2 in Supplemental Material [60] have been recorded using the same dipole set and $R=10 \mathrm{~mm}$. By scanning the frequency range between 2.5 and $5.25 \mathrm{GHz}$, we tune the factor $k R$ from 0.5 to 1.1 . We stress again that the theoretical results in Fig. 2 are not a fit to the experimental data-there is no free parameter. Only the relative phase is adjusted for two frequencies ( 3 and $4.8 \mathrm{GHz}$ ) to find the correct balance between CDR and CLS. For other frequencies, the phase is linearized and follows a linear behavior with the frequency and $k R$. Considering the complexity of the processes we are considering, and the finite sizes of the dipoles and mirrors in our experiments, we find excellent agreement between the experimental data and the results derived from theory. All the main theoretical features for 

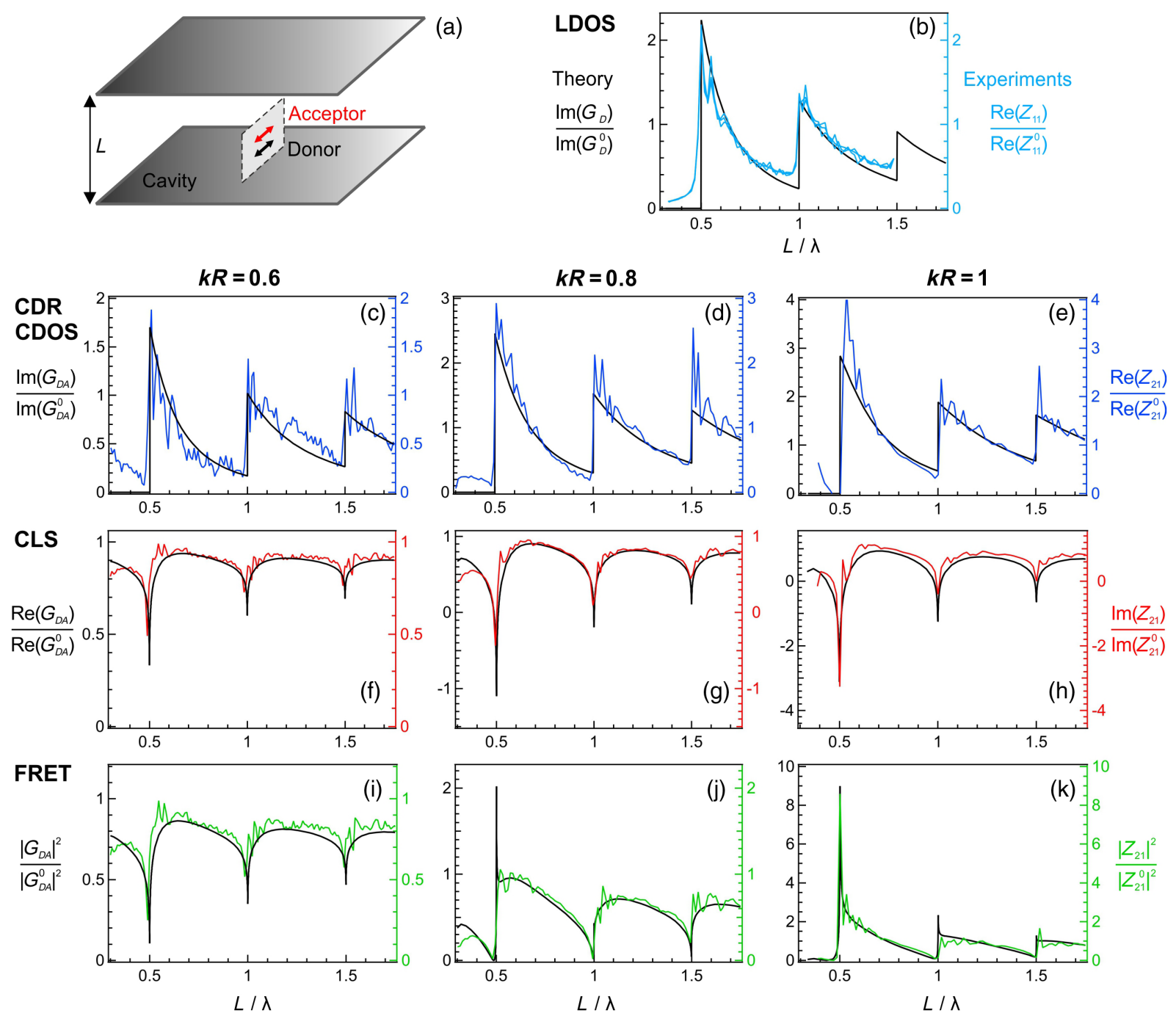

FIG. 2. Complex-valued Green function in a planar cavity compared to that in vacuum. (a) Configuration under study: the source donor dipole is located at $10 \mathrm{~mm}$ from the nearest mirror and is parallel to the mirror plane. The acceptor dipole is parallel to the donor dipole at $R=10 \mathrm{~mm}$ distance. The frequency varies between 2.5 and $5.25 \mathrm{GHz}$. (b) Measured LDOS enhancement versus cavity length $L$ at $5 \mathrm{GHz}$. The thin colored curves are measurements of the impedance $Z_{11}$, whereas the thick black curve is the analytical prediction. (c)-(e) CDR or CDOS enhancement over vacuum versus cavity length for different $k R$ values (indicated on top). The colored thin curves indicate measurements, whereas the thick black curves are the analytic results. (f)-(h) CLS enhancement, i.e., enhancement of the real part, over vacuum. (i)-(k) FRET enhancement, i.e., enhancement of the square modulus, over vacuum. These measurements provide a complete characterization of the complex Green function in a planar cavity.

point dipoles are confirmed experimentally. These features include the resonances at lengths multiples of $\lambda / 2$, the cutoff for the CDR at cavity lengths below $\lambda / 2$, the evolution of the curves with $L$, and the relative evolutions of the enhancement factors as $k R$ increases.

For a lossless cavity, it is known that only propagative radiative modes contribute to the CDR and the imaginary part of the Green function [37]. This leads to a complete cutoff for the CDR and CDOS when the cavity length is below $\lambda / 2$ and no propagative mode exists, in close analogy to the LDOS [Fig. 2(b)]. In contrast, the real part of the Green function depends on both propagating and evanescent cavity modes $[37,61,62]$. The evanescent modes have no cutoff and so the CLS remains finite for very short cavities. As the FRET depends on the square modulus of the Green function and combines contributions from both the real and imaginary part of $\stackrel{\leftrightarrow}{\boldsymbol{G}}\left(\boldsymbol{r}_{A}, \boldsymbol{r}_{D}\right)$, it features dips or peaks depending on the distance between dipoles which determines the relative strengths of the real and the imaginary parts of $\stackrel{\leftrightarrow}{\boldsymbol{G}}\left(\boldsymbol{r}_{A}, \boldsymbol{r}_{D}\right)$ (Fig. 1) [44].

Altogether, the experimental data in Fig. 2 and Figs. S1-S3 [60] demonstrate that the complex-valued Green function can be fully characterized inside a resonant planar cavity, yielding information on its real and imaginary parts, as well as its square modulus. For low $k R$ 
values, the signal-to-noise ratio drops as the dipole size becomes much smaller than the wavelength. This primarily affects measurements of $\operatorname{Re}\left(Z_{21}\right)$, which is a resistance and is more sensitive to noise than $\operatorname{Im}\left(Z_{21}\right)$, which corresponds to an inductance or capacitance. Though longer dipoles could partly solve this issue, this has limited scope as dipoles are ideally infinitely small. Additional effects due to the finite size of the mirrors or their lack of perfect planarity or parallelism (for very large mirrors of dimensions exceeding $1 \mathrm{~m}$ ) could also affect our measurements, imposing a trade-off in the system configuration.

Specific projections of the Green dyadic can be chosen by adjusting the orientations of the two dipoles. In a single experimental run, this methodology allows exploration of the influence of a resonant planar cavity on cooperative effects such as CDR, CDOS, CLS, and FRET. We find that the CDR and CDOS follow a trend very close to the LDOS evolution inside the cavity. On the other hand, the CLS and FRET exhibit sharp resonances at cavity lengths which are integer multiples of $\lambda / 2$. Consistent with predictions based on the analytic expression for the Green function, the cavity enhancement is more pronounced for dipole-dipole separations exceeding $0.1 \lambda$ (corresponding to $k R>0.7$ ) and increase with $R$. For small dipole-dipole distances below $0.05 \lambda$ (corresponding to $k R<0.3$ ), we find that the RDDI is marginally affected by the cavity, leading to minor variations of the CDR, CLS, or FRET. This detailed information of RDDI inside a resonant cavity is important to help the design of future experiments aiming at maximizing the cooperative decay rate or the cooperative Lamb shift [18-21].

Having validated our experimental approach for a configuration for which the complete analytic form of the Green function is known, we now turn to a case for which no such analytic solution exists. We realize this configuration by tilting one mirror forming the cavity by an angle $\theta$ [Fig. 3(a)]. All the other parameters are kept identical to that in Fig. 2. Owing to the open configuration and the large size of the mirrors, which exceed $5 \lambda$, numerical simulations of this tilted cavity require several hours of computation time even on high-performance computers (it took us about $10 \mathrm{~h}$ on an Intel Core i76700 processor running at $3.4 \mathrm{GHz}$ with $40 \mathrm{~GB}$ of ram). In contrast, the measurement of the complete $Z$ matrix takes only a few seconds, and a broad range of cavity lengths and emission frequencies can be scanned within a few minutes. Figures 3(b)-3(d) compare the experimental results with numerical simulations for the real and imaginary parts of the Green function with respect to the vacuum reference. The data for two tilt angles of $5^{\circ}$ and $10^{\circ}$ are compared to the parallel cavity case. As seen in both the experimental and simulation data, increasing the mirror angle broadens

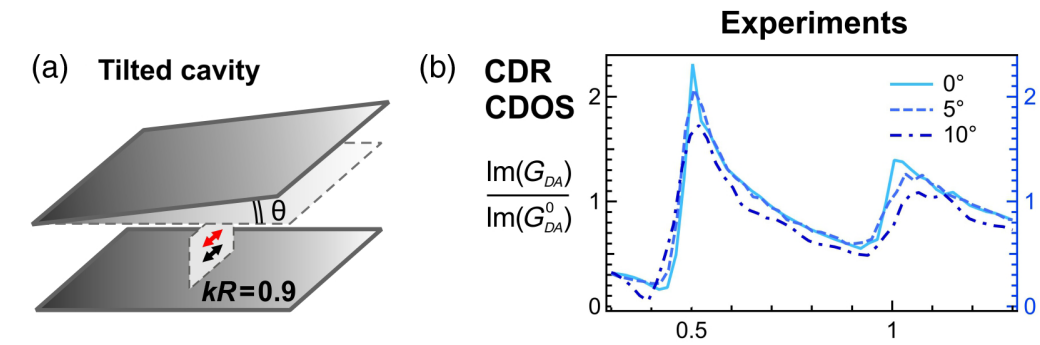

(c)
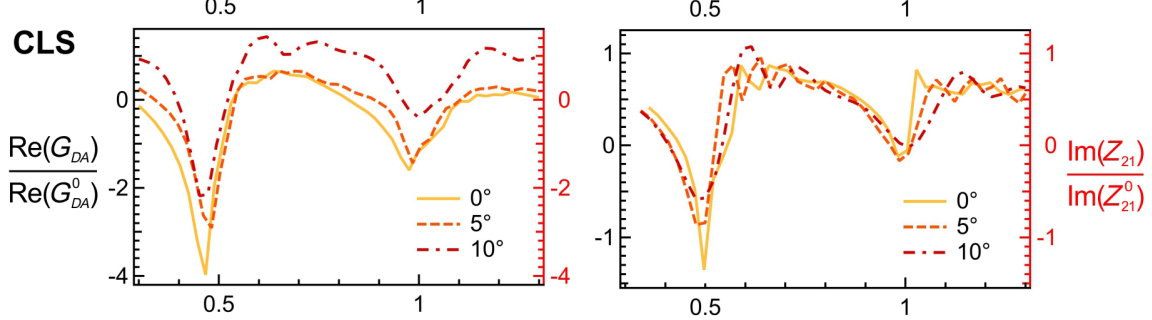

(d)
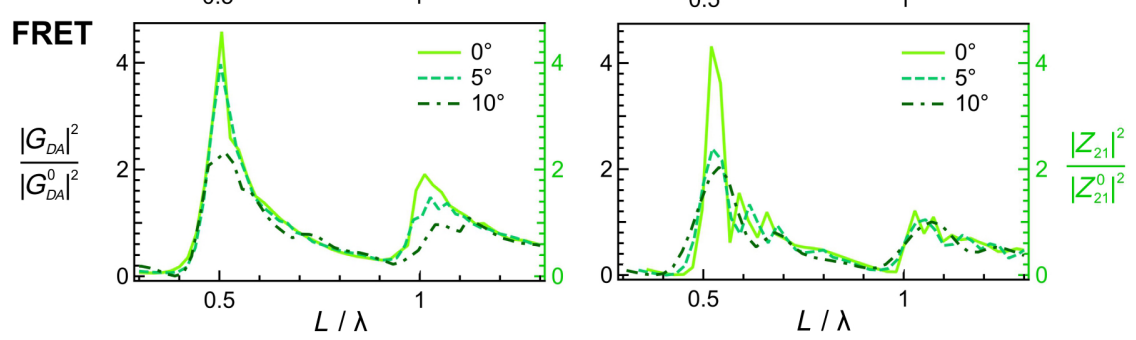

FIG. 3. Effect of a nonplanar cavity on the Green function, corresponding to a case for which no analytic solution exists. (a) One mirror forming the cavity is tilted by an angle $\theta$; the rest of the configuration is similar to Fig. 2 . The distance between the dipoles is $k R=0.9$. (b) CDR-CDOS, (c) CLS, and (d) FRET enhancement factors versus the cavity length for different tilt angles $\theta$. The left-hand column shows the experimental results, whereas the right-hand column presents the numerical simulation results using the finite domain time difference method (CST Studio suite 2019). 
the cavity resonance, reducing its quality factor. Both the real and imaginary parts of the Green function are affected, but the real part of $\stackrel{\leftrightarrow}{\boldsymbol{G}}$ (leading to the CLS and to a large extent to FRET) appears to be comparatively more affected by the lack of parallelism than the imaginary part (corresponding to the CDR and CDOS). We relate this to the fact that the real part of $\stackrel{\leftrightarrow}{\boldsymbol{G}}$ diverges while $R$ tends toward 0 , while the imaginary part of $\stackrel{\leftrightarrow}{\boldsymbol{G}}$ is asymptotically constant when $R \rightarrow 0$ [Figs. 1(a) and 1(b)]. The good agreement between the experimental data and the numerical simulation results further confirm the validity of our approach.

To further demonstrate the capabilities of our technique and strengthen the link with studies in the optical regime, we investigate the role of a dielectric subwavelength scatterer in mediating the resonant dipole-dipole interaction and enhancing the energy transfer (Fig. 4). Such a capacity has attracted much interest in the visible regime, but owing to the high difficulty of experiments at optical frequencies, the works performed so far mainly focused on theoretical and numerical studies [63-73]. Experimental attempts to address this problem in optics have led to inconsistent results showing FRET enhancement in some cases [74,75] but not in others [76,77]. By performing our experiments at radio frequencies, where the $10^{5}$ larger wavelength enables positioning of the dipole source and receiver with deep subwavelength precision and excellent control on the mutual orientation, we take full advantage of the scalability of our approach.

Figure 4(a) illustrates our experiment: two parallel dipoles are placed on opposite faces of a dielectric cube of $16 \mathrm{~mm}$ side length (corresponding to $\lambda / 5$ ). The cube material is chosen to mimic the properties of silicon in the optical near-infrared regime with a dielectric permittivity of $16+i 0.25$ (Eccostock HIK, $\mathrm{K}=16$ ). The scattering (a)

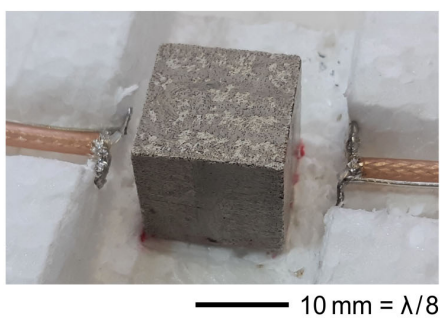

(c)

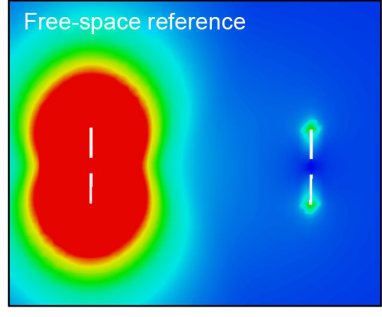

(d)

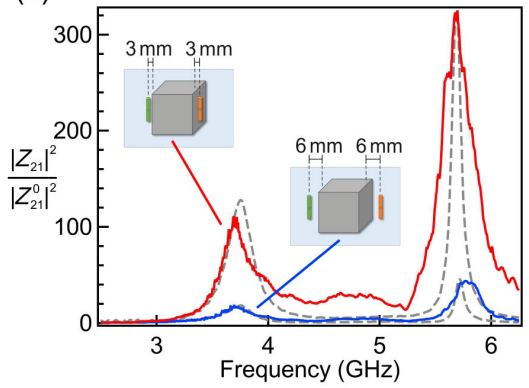

(b)

(e)
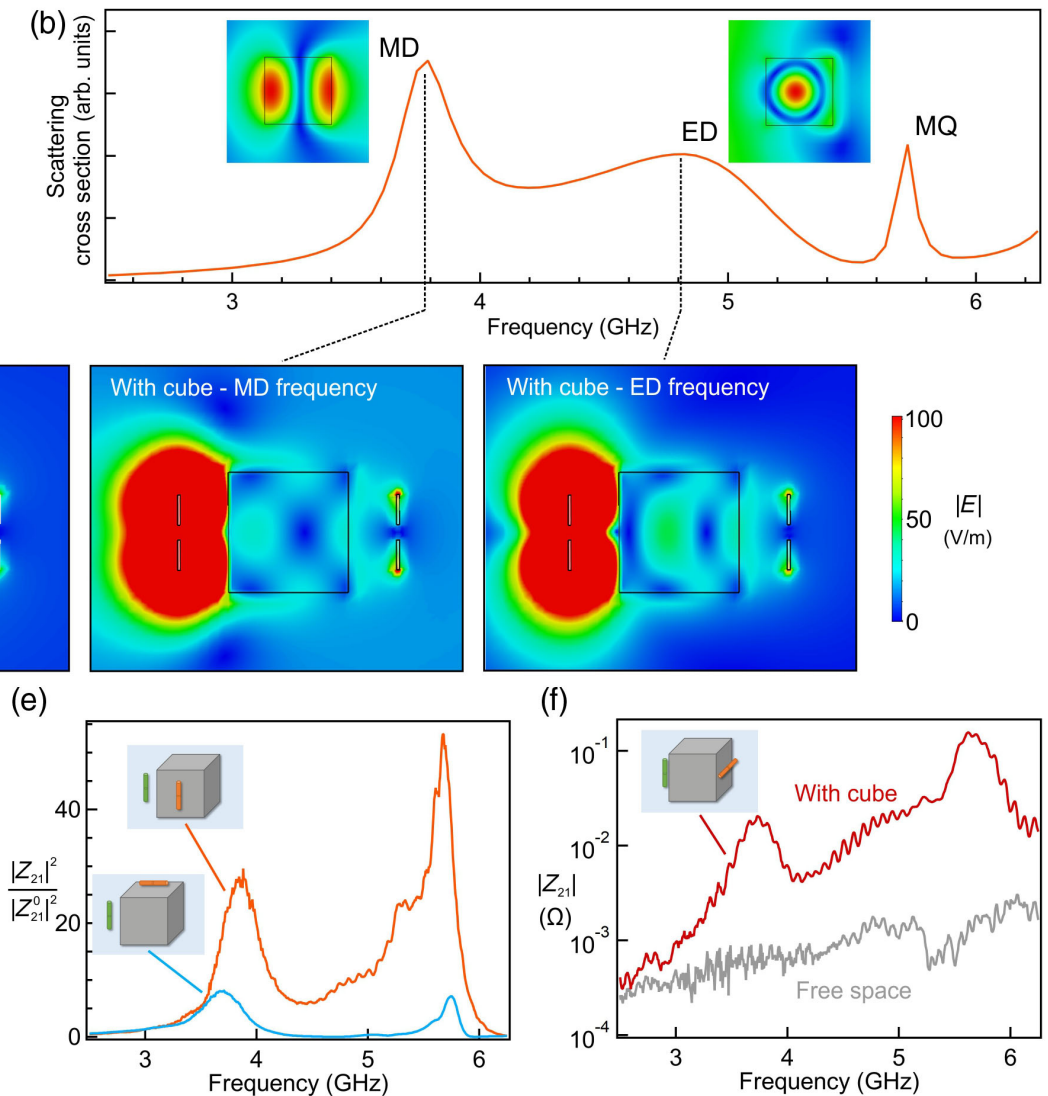

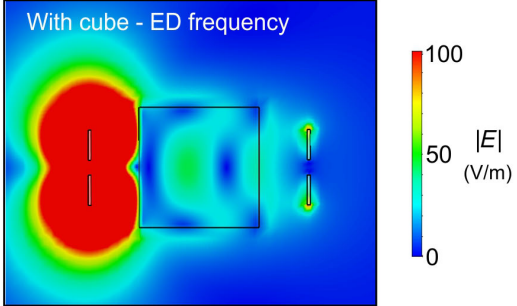

(f)

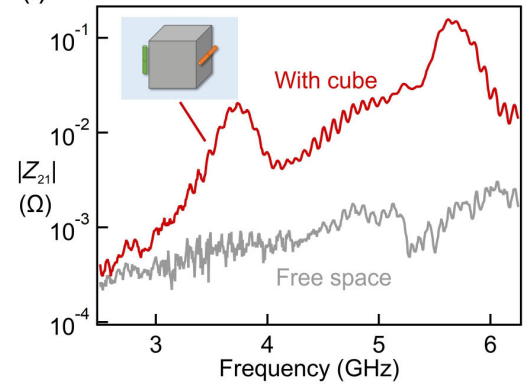

FIG. 4. Dipole-dipole interaction mediated by a dielectric cube scatterer. (a) Schematic of the experiment: the source dipole is on the left, the receiving dipole is on the right, and a $16 \mathrm{~mm}$ cube of dielectric permittivity 16 is placed between them. The distance between the dipole and the surface of the cube is $3 \mathrm{~mm}(\lambda / 26)$ or $6 \mathrm{~mm}(\lambda / 13)$. (b) Numerical simulations of the scattering cross section; the magnetic dipole (MD), electric dipole (ED), and magnetic quadrupole (MQ) resonances are clearly visible. The insets show the electric field amplitude at the MD and ED resonance frequencies, respectively, for plane wave illumination incoming from the left. (c) Numerical simulations of the electric field amplitude in the presence and absence of the dielectric cube at the MD or ED resonance frequencies. The dipoles are placed $6 \mathrm{~mm}$ from the cube. (d)-(f) Measurements of the FRET enhancement for different configurations. In (d) the dipoles are parallel and on opposite faces of the cube with 3 and $6 \mathrm{~mm}$ distances to the cube, respectively. The gray dashed lines show the result of numerical simulations. In (e) the dipoles are on adjacent faces of the cubes with $6 \mathrm{~mm}$ distance. In (f) the dipoles have a perpendicular orientation to each other. The dipole distance to the cube surface is again $6 \mathrm{~mm}$. 
spectrum in Fig. 4(b) exhibits several resonances corresponding, respectively, to the magnetic dipole (MD), electric dipole (ED), and magnetic quadrupole (MQ) modes of the cube [78,79]. The magnetic modes lead to a current loop inside the cube and, hence, the electric field has a maximum amplitude near the cube surface. In contrast, for the electric dipole mode the electric field has a maximum amplitude near the center of the cube. Therefore, based on the electric field distributions, we expect a maximum coupling between the two dipoles at the magnetic MD and MQ modes, and weak coupling at the ED mode. Numerical simulations including both dipoles confirm this intuition [Fig. 4(c)]. At the MD resonant frequency, the electric field amplitude at the position of the second dipole is enhanced by a factor of 4, whereas at the ED frequency the amplitude is roughly the same as in free space.

Figure 4(d) shows our experimental results corresponding to the configuration in Fig. 4(c). Indeed, we find a peak enhancement of the energy transfer between dipoles at the $\mathrm{MD}$ and MQ resonant frequencies, and a marginal increase at the ED frequency. The enhancement appears larger at the MQ frequency as the radiation efficiency increases with frequency, but the 10-mm-long dipole source can now no longer be considered to be a point source. We also observe a clear dependence on the distance between the dipoles and the cube: for short distances $(3 \mathrm{~mm}$ versus $6 \mathrm{~mm}$ ), the coupling is increased, leading to an energy transfer enhancement of over $100 \times$. All these different features are consistent with our numerical simulations [dashed gray lines in Fig. 4(d)]. While optical experiments have led to contradictory results, showing FRET enhancement $[74,75]$ and inhibition [76,77], our radio frequency data provide a clear experimental demonstration of the influence of the various resonance modes in a dielectric scatterer to mediate the energy transfer between two dipoles, confirming the trend expected from theoretical investigations [63-73].

A strength of our approach is that it enables the measurement of a large variety of configurations. In Fig. 4(e), we explore the relative position of the two dipoles on adjacent faces of the cube. When the acceptor dipole is positioned in the plane corresponding to the main emission lobe of the donor dipole [plane perpendicular to the dipole axis, orange curve in Fig. 4(e)], the energy transfer is maximized. In contrast, when the acceptor dipole is positioned in the plane parallel with the donor dipole axis, the FRET enhancement is lower as the donor energy is directed mainly elsewhere. This helps to better understand the inconclusive results in the optical regime [74-77], where the orientation of the emitters is difficult to control.

Another remarkable feature is that the cube enables energy transfer between perpendicular dipoles [Fig. 4(f)]. For point dipoles in free space, there is theoretically no energy transfer when the dipoles are set perpendicular to each other [1]. In our free-space experiments, the residual mutual impedance is nonzero due to the finite size of the dipoles, but the recorded $0.6 \mathrm{~m} \Omega$ impedance is close to the limit of sensitivity of our apparatus. In the presence of the dielectric cube, the FRET enhancement $\left|Z_{21}\right|^{2} /\left|Z_{21}^{0}\right|^{2}$ exceeds $1100 \times$. This large enhancement value is mainly due to the vanishing reference, but it confirms that energy transfer indeed occurs. In the presence of the cube, the electric field from the source now features components along all the three directions of space, and these new field components open the possibility to transfer energy to the acceptor dipole. A similar trend was observed in optical experiments between perpendicular dipoles in the presence of a nanostructure [80], yet the absence of position control between the FRET dipoles and the nanostructure complicated the analysis. Lastly, we use the different datasets in Figs. 4(d)-4(f) to check that the reciprocity condition $Z_{21}=Z_{12}$ is verified in our experiments (Fig. S4 in Supplemental Material [60]]).

\section{CONCLUSION}

To conclude, we have shown that the mutual impedance $Z_{21}$ of a two-port network is directly proportional to the Green function $\stackrel{\leftrightarrow}{\boldsymbol{G}}$ projected on the dipole's axis. This enables a complete characterization of both the real and imaginary parts of the Green function in an arbitrary environment, and provides a unique way to investigate resonant dipole-dipole interactions and cooperative effects (CDR, CDOS, CLS, and FRET). We demonstrate the effectiveness of this approach by measuring the complete Green function inside a planar cavity of parallel and nonparallel mirrors and with a dielectric cube scatterer. Providing a deeper link between microwave engineering and classical and quantum electrodynamics serves not only to unify the descriptions of cooperative dipole-dipole interactions, it also gives a practical tool to fully characterize complex photonic systems using a single straightforward approach. Given the ubiquitous nature of the Green function in electromagnetism, from optics to radio frequencies, we believe that the applications of this work could concern a very wide field. Beyond electromagnetism, it can inspire analogies in other areas of wave physics, such as acoustics, for instance.

\section{ACKNOWLEDGMENTS}

This research was conducted within the context of the International Associated Laboratory "ALPhFA: Associated Laboratory for Photonics between France and Australia." This work has received funding from the European Union's Horizon 2020 Research and Innovation programme under Grant Agreement No. 736937, from the Agence Nationale de la Recherche (ANR) under Grant Agreement No. ANR-17-CE09-0026-01, and from Excellence Initiative of Aix-Marseille University-A*MIDEX, a French "Investissements d'Avenir" program. 


\section{APPENDIX: EXPERIMENTAL SETUP}

We work in the frequency range from 2.5 to $5.25 \mathrm{GHz}$, corresponding to wavelengths from 12 to $5.7 \mathrm{~cm}$. The dipoles are built from a RG-316 coaxial cable. Each dipole has an $11 \mathrm{~mm}$ total length, $1 \mathrm{~mm}$ width, $1 \mathrm{~mm}$ thickness, and a $2 \mathrm{~mm}$ gap between the branches. The RG-316 coaxial cable has a $50 \Omega$ impedance and a $6 \mathrm{~cm}$ length. A $1.75-\mathrm{cm}-$ long Pawsey stub balun $(\lambda / 4$ at $4.25 \mathrm{GHz})$ connects the arm of the dipole from the inner conductor to the shield of the cable. The balun aims to reduce the return current on the outside of the coaxial cable shield and improve the precision of the measurements. The dipole resonance frequency is $7.42 \mathrm{GHz}$ and remains well outside the frequency range used for our experiments. This ensures that the dipoles can still be considered as nearly punctual in our experiments. The two dipoles are separated by a fixed distance of $10 \mathrm{~mm}$, and are held on a foam spacer of approximately 1.08 dielectric permittivity.

The dipoles are connected to a vector network analyzer (VNA, Anritsu model MS2036C) through K-type cables of $18 \mathrm{GHz}$ bandwidth. The VNA is calibrated over the complete frequency bandwidth taking into account the presence of the K-type cables. The VNA records the $S$ and the $Z$ matrix. Each data point is obtained after passing through a $500 \mathrm{~Hz}$ filter without averaging. The presence of the $6 \mathrm{~cm}$ of RG-316 coaxial cable connecting the dipoles leads to an additional phase delay which is not accounted for in the VNA calibration process. In the calculation of the $Z$ matrix data, we compensate for this additional phase term by adjusting the phase at two selected frequencies ( 3 and 4.8 $\mathrm{GHz}$ ). The phase for the other frequencies is then linearly extrapolated from this adjustment.

The cavity is formed by two mirrors of dimensions $60 \times 60 \mathrm{~cm}^{2}$. For the parallel configuration (Fig. 2), the parallelism between the mirrors is checked to be better than $0.5^{\circ}$. All the experiments reported here are performed with the source donor dipole positioned at $10 \mathrm{~mm}$ from the nearest mirror. This configuration allows us to break the symmetry to monitor resonances occurring at $L=\lambda$ and $L=2 \lambda$. We ensured that our conclusions are not significantly changed as compared to the configuration where the donor dipole is located exactly in the cavity center. The cavity length $L$ is scanned by moving one mirror with a motorized translation stage.

[1] L. Novotny and B. Hecht, Principles of Nano-Optics (Cambridge University Press, Cambridge, England, 2012).

[2] P. Bharadwaj, B. Deutsch, and L. Novotny, Optical Antennas, Adv. Opt. Photonics 1, 438 (2009).

[3] R. Carminati, A. Cazé, D. Cao, F. Peragut, V. Krachmalnicoff, R. Pierrat, and Y. De Wilde, Electromagnetic Density of States in Complex Plasmonic Systems, Surf. Sci. Rep. 70, 1 (2015).
[4] W. L. Barnes, S. A. R. Horsley, and W. L. Vos, Classical Antennae, Quantum Emitters, and Densities of Optical States, J. Opt. 22, 073501 (2020).

[5] P. Lodahl, A. F. van Driel, I. S. Nikolaev, A. Irman, K. Overgaag, D. Vanmaekelbergh, and W. L. Vos, Controlling the Dynamics of Spontaneous Emission from Quantum Dots by Photonic Crystals, Nature (London) 430, 654 (2004).

[6] A. Cazé, R. Pierrat, and R. Carminati, Spatial Coherence in Complex Photonic and Plasmonic Systems, Phys. Rev. Lett. 110, 063903 (2013).

[7] J. de Rosny and M. Davy, Green's Function Retrieval and Fluctuations of Cross Density of States in MultipleScattering Media, Europhys. Lett. 106, 54004 (2014).

[8] R. H. Dicke, Coherence in Spontaneous Radiation Processes, Phys. Rev. 93, 99 (1954).

[9] H. T. Dung, L. Knöll, and D.-G. Welsch, Resonant DipoleDipole Interaction in the Presence of Dispersing and Absorbing Surroundings, Phys. Rev. A 66, 063810 (2002).

[10] C. L. Cortes and Z. Jacob, Super-Coulombic Atom-Atom Interactions in Hyperbolic Media, Nat. Commun. 8, 1 (2017).

[11] R. Friedberg, S. R. Hartmann, and J. T. Manassah, Frequency Shifts in Emission and Absorption by Resonant Systems ot Two-Level Atoms, Phys. Rep. 7, 101 (1973).

[12] P. Andrew and W. L. Barnes, Förster Energy Transfer in an Optical Microcavity, Science 290, 785 (2000).

[13] C. Blum, N. Zijlstra, A. Lagendijk, M. Wubs, A. P. Mosk, V. Subramaniam, and W. L. Vos, Nanophotonic Control of the Förster Resonance Energy Transfer Efficiency, Phys. Rev. Lett. 109, 203601 (2012).

[14] P. Ghenuche, J. de Torres, S. B. Moparthi, V. Grigoriev, and J. Wenger, Nanophotonic Enhancement of the Förster Resonance Energy-Transfer Rate with Single Nanoapertures, Nano Lett. 14, 4707 (2014).

[15] P. Goy, J. M. Raimond, M. Gross, and S. Haroche, Observation of Cavity-Enhanced Single-Atom Spontaneous Emission, Phys. Rev. Lett. 50, 1903 (1983).

[16] H. Walther, B. T. H. Varcoe, B.-G. Englert, and T. Becker, Cavity Quantum Electrodynamics, Rep. Prog. Phys. 69, 1325 (2006).

[17] M. O. Scully, Collective Lamb Shift in Single Photon Dicke Superradiance, Phys. Rev. Lett. 102, 143601 (2009).

[18] R. Röhlsberger, K. Schlage, B. Sahoo, S. Couet, and R. Rüffer, Collective Lamb Shift in Single-Photon Superradiance, Science 328, 1248 (2010).

[19] J. Keaveney, A. Sargsyan, U. Krohn, I. G. Hughes, D. Sarkisyan, and C. S. Adams, Cooperative Lamb Shift in an Atomic Vapor Layer of Nanometer Thickness, Phys. Rev. Lett. 108, 173601 (2012).

[20] J. Kim, D. Yang, S. Oh, and K. An, Coherent Single-Atom Superradiance, Science 359, 662 (2018).

[21] T. Peyrot, Y. R. P. Sortais, A. Browaeys, A. Sargsyan, D. Sarkisyan, J. Keaveney, I. G. Hughes, and C. S. Adams, Collective Lamb Shift of a Nanoscale Atomic Vapor Layer within a Sapphire Cavity, Phys. Rev. Lett. 120, 243401 (2018).

[22] F. Borjans, X. G. Croot, X. Mi, M. J. Gullans, and J.R. Petta, Resonant Microwave-Mediated Interactions between Distant Electron Spins, Nature (London) 577, 195 (2020). 
[23] L. Novotny and N. van Hulst, Antennas for Light, Nat. Photonics 5, 83 (2011).

[24] C. L. Cortes, W. Newman, S. Molesky, and Z. Jacob, Quantum Nanophotonics Using Hyperbolic Metamaterials, J. Opt. 14, 063001 (2012).

[25] V. N. Pustovit and T. V. Shahbazyan, Cooperative Emission of Light by an Ensemble of Dipoles near a Metal Nanoparticle: The Plasmonic Dicke Effect, Phys. Rev. Lett. 102, 077401 (2009).

[26] W. D. Newman, C. L. Cortes, A. Afshar, K. Cadien, A. Meldrum, R. Fedosejevs, and Z. Jacob, Observation of Long-Range Dipole-Dipole Interactions in Hyperbolic Metamaterials, Sci. Adv. 4, eaar5278 (2018).

[27] K. G. Cognée, H. M. Doeleman, P. Lalanne, and A. F. Koenderink, Cooperative Interactions between NanoAntennas in a High-Q Cavity for Unidirectional Light Sources, Light Sci. Appl. 8, 115 (2019).

[28] R. Carminati, R. Pierrat, J. de Rosny, and M. Fink, Theory of the Time Reversal Cavity for Electromagnetic Fields, Opt. Lett. 32, 3107 (2007).

[29] J. de Rosny, G. Lerosey, and M. Fink, Theory of Electromagnetic Time-Reversal Mirrors, IEEE Trans. Antennas Propag. 58, 3139 (2010).

[30] O. I. Lobkis and R. L. Weaver, On the Emergence of the Green's Function in the Correlations of a Diffuse Field, J. Acoust. Soc. Am. 110, 3011 (2001).

[31] K. Wapenaar, Retrieving the Elastodynamic Green's Function of an Arbitrary Inhomogeneous Medium by Cross Correlation, Phys. Rev. Lett. 93, 254301 (2004).

[32] M. Farhat, S. Guenneau, and S. Enoch, Ultrabroadband Elastic Cloaking in Thin Plates, Phys. Rev. Lett. 103, 024301 (2009).

[33] G. Chen, J. Stang, and M. Moghaddam, Numerical Vector Green's Function for S-Parameter Measurement With Waveport Excitation, IEEE Trans. Antennas Propag. 65, 3645 (2017).

[34] H. C. Chen, Theory of Electromagnetic Waves: A CoordinateFree Approach (McGraw-Hill, New York, 1983).

[35] J. E. Sipe, New Green-Function Formalism for Surface Optics, J. Opt. Soc. Am. B 4, 481 (1987).

[36] D. P. Fussell, R. C. McPhedran, and C. Martijn de Sterke, Three-Dimensional Green's Tensor, Local Density of States, and Spontaneous Emission in Finite Two-Dimensional Photonic Crystals Composed of Cylinders, Phys. Rev. E 70, 066608 (2004).

[37] T. Kobayashi, Q. Zheng, and T. Sekiguchi, Resonant Dipole-Dipole Interaction in a Cavity, Phys. Rev. A 52, 2835 (1995).

[38] V. Krachmalnicoff, E. Castanié, Y. De Wilde, and R. Carminati, Fluctuations of the Local Density of States Probe Localized Surface Plasmons on Disordered Metal Films, Phys. Rev. Lett. 105, 183901 (2010).

[39] V. Krachmalnicoff, D. Cao, A. Cazé, E. Castanié, R. Pierrat, N. Bardou, S. Collin, R. Carminati, and Y.D. Wilde, Towards a Full Characterization of a Plasmonic Nanostructure with a Fluorescent near-Field Probe, Opt. Express 21, 11536 (2013).

[40] D. Cao, A. Cazé, M. Calabrese, R. Pierrat, N. Bardou, S. Collin, R. Carminati, V. Krachmalnicoff, and Y. De Wilde, Mapping the Radiative and the Apparent Nonradiative
Local Density of States in the Near Field of a Metallic Nanoantenna, ACS Photonics 2, 189 (2015).

[41] F. J. G. de Abajo and M. Kociak, Probing the Photonic Local Density of States with Electron Energy Loss Spectroscopy, Phys. Rev. Lett. 100, 106804 (2008).

[42] A. Hörl, A. Trügler, and U. Hohenester, Tomography of Particle Plasmon Fields from Electron Energy Loss Spectroscopy, Phys. Rev. Lett. 111, 076801 (2013).

[43] A. Hörl, A. Trügler, and U. Hohenester, Full ThreeDimensional Reconstruction of the Dyadic Green Tensor from Electron Energy Loss Spectroscopy of Plasmonic Nanoparticles, ACS Photonics 2, 1429 (2015).

[44] K. Rustomji, M. Dubois, B. Kuhlmey, C. M. de Sterke, S. Enoch, R. Abdeddaim, and J. Wenger, Direct Imaging of the Energy-Transfer Enhancement between Two Dipoles in a Photonic Cavity, Phys. Rev. X 9, 011041 (2019).

[45] M. Davy, M. Fink, and J. de Rosny, Green's Function Retrieval and Passive Imaging from Correlations of Wideband Thermal Radiations, Phys. Rev. Lett. 110, 203901 (2013).

[46] K. Rustomji, R. Abdeddaim, C. M. de Sterke, B. Kuhlmey, and S. Enoch, Measurement and Simulation of the Polarization-Dependent Purcell Factor in a Microwave Fishnet Metamaterial, Phys. Rev. B 95, 035156 (2017).

[47] M. Baraclough, S. S. Seetharaman, I. R. Hooper, and W. L. Barnes, Metamaterial Analogues of Molecular Aggregates, ACS Photonics 6, 3003 (2019).

[48] J. B. Pendry, L. Martín-Moreno, and F. J. Garcia-Vidal, Mimicking Surface Plasmons with Structured Surfaces, Science 305, 847 (2004).

[49] A. P. Hibbins, B. R. Evans, and J. R. Sambles, Experimental Verification of Designer Surface Plasmons, Science $\mathbf{3 0 8}$, 670 (2005).

[50] M. Hopmeier, W. Guss, M. Deussen, E. O. Göbel, and R. F. Mahrt, Enhanced Dipole-Dipole Interaction in a Polymer Microcavity, Phys. Rev. Lett. 82, 4118 (1999).

[51] A. Konrad, M. Metzger, A. M. Kern, M. Brecht, and A. J. Meixner, Controlling the Dynamics of Förster Resonance Energy Transfer inside a Tunable Sub-Wavelength FabryPérot-Resonator, Nanoscale 7, 10204 (2015).

[52] D. M. Coles, N. Somaschi, P. Michetti, C. Clark, P. G. Lagoudakis, P. G. Savvidis, and D. G. Lidzey, PolaritonMediated Energy Transfer between Organic Dyes in a Strongly Coupled Optical Microcavity, Nat. Mater. 13, 712 (2014).

[53] X. Zhong, T. Chervy, S. Wang, J. George, A. Thomas, J. A. Hutchison, E. Devaux, C. Genet, and T. W. Ebbesen, Non-Radiative Energy Transfer Mediated by Hybrid Light-Matter States, Angew. Chem. Int. Ed. 55, 6202 (2016).

[54] C. A. Balanis, Antenna Theory: Analysis and Design, 3rd ed. (John Wiley \& Sons, New York, 2005).

[55] S. A. Tretyakov, S. Maslovski, and P. A. Belov, An Analytical Model of Metamaterials Based on Loaded Wire Dipoles, IEEE Trans. Antennas Propag. 51, 2652 (2003).

[56] J.-J. Greffet, M. Laroche, and F. Marquier, Impedance of a Nanoantenna and a Single Quantum Emitter, Phys. Rev. Lett. 105, 117701 (2010).

[57] A. E. Krasnok, A. P. Slobozhanyuk, C. R. Simovski, S. A. Tretyakov, A. N. Poddubny, A. E. Miroshnichenko, 
Y. S. Kivshar, and P. A. Belov, An Antenna Model for the Purcell Effect, Sci. Rep. 5, 12956 (2015).

[58] J.-K. Hwang, H.-Y. Ryu, and Y.-H. Lee, Spontaneous Emission Rate of an Electric Dipole in a General Microcavity, Phys. Rev. B 60, 4688 (1999).

[59] F. B. Seeley, J. E. Alexander, R. W. Connatser, J. S. Conway, and J. P. Dowling, Dipole Radiators in a Cavity: A Radio Frequency Analog for the Modification of Atomic Spontaneous Emission Rates between Mirrors, Am. J. Phys. 61, 545 (1993).

[60] See Supplemental Material at http://link.aps.org/supplemental/ 10.1103/PhysRevX.11.021004 for supplementary data for different $k R$ values, influence of the dipole-dipole separation on the cavity enhancement, and reciprocity check.

[61] M. Wubs, L. G. Suttorp, and A. Lagendijk, Multipole Interaction between Atoms and Their Photonic Environment, Phys. Rev. A 68, 013822 (2003).

[62] M. Wubs, L. G. Suttorp, and A. Lagendijk, MultipleScattering Approach to Interatomic Interactions and Superradiance in Inhomogeneous Dielectrics, Phys. Rev. A 70, 053823 (2004).

[63] L.-Y. Hsu, W. Ding, and G. C. Schatz, Plasmon-Coupled Resonance Energy Transfer, J. Phys. Chem. Lett. 8, 2357 (2017).

[64] A. O. Govorov, J. Lee, and N. A. Kotov, Theory of PlasmonEnhanced Förster Energy Transfer in Optically Excited Semiconductor and Metal Nanoparticles, Phys. Rev. B 76, 125308 (2007).

[65] H. Y. Xie, H. Y. Chung, P. T. Leung, and D. P. Tsai, Plasmonic Enhancement of Förster Energy Transfer between Two Molecules in the Vicinity of a Metallic Nanoparticle: Nonlocal Optical Effects, Phys. Rev. B 80, 155448 (2009).

[66] V. N. Pustovit and T. V. Shahbazyan, Resonance Energy Transfer near Metal Nanostructures Mediated by Surface Plasmons, Phys. Rev. B 83, 085427 (2011).

[67] R. Vincent and R. Carminati, Magneto-Optical Control of Förster Energy Transfer, Phys. Rev. B 83, 165426 (2011).

[68] J. A. Gonzaga-Galeana and J. R. Zurita-Sánchez, A Revisitation of the Förster Energy Transfer near a Metallic Spherical Nanoparticle: (1) Efficiency Enhancement or Reduction? (2) The Control of the Förster Radius of the Unbounded Medium. (3) The Impact of the Local Density of States, J. Chem. Phys. 139, 244302 (2013).

[69] R. Chang, P. T. Leung, and D. P. Tsai, Effects of Gain Medium on the Plasmonic Enhancement of Forster
Resonance Energy Transfer in the Vicinity of a Metallic Particle or Cavity, Opt. Express 22, 27451 (2014).

[70] W. Ding, L.-Y. Hsu, and G. C. Schatz, Plasmon-Coupled Resonance Energy Transfer: A Real-Time Electrodynamics Approach, J. Chem. Phys. 146, 064109 (2017).

[71] V. N. Pustovit, A. M. Urbas, and T. V. Shahbazyan, Energy Transfer in Plasmonic Systems, J. Opt. 16, 114015 (2014).

[72] Y.-C. Yu, J.-M. Liu, C.-J. Jin, and X.-H. Wang, PlasmonMediated Resonance Energy Transfer by Metallic Nanorods, Nanoscale Res. Lett. 8, 209 (2013).

[73] Y. Jeong and G. C. Schatz, Enhancement and Suppression of Resonance Energy Transfer near Metal Nanoparticles, J. Phys. Chem. C 124, 20589 (2020).

[74] N. Aissaoui, K. Moth-Poulsen, M. Käll, P. Johansson, L. M. Wilhelmsson, and B. Albinsson, FRET Enhancement Close to Gold Nanoparticles Positioned in DNA Origami Constructs, Nanoscale 9, 673 (2017).

[75] N. T. Anderson, S. Ren, J. Chao, P. H. Dinolfo, and X. Wang, Exploiting Plasmon-Mediated Energy Transfer to Enhance End-to-End Efficiency in a DNA Origami Energy Transfer Array, ACS Appl. Nano Mater. 2, 5563 (2019).

[76] J. Bohlen, Á. Cuartero-González, E. Pibiri, D. Ruhlandt, A. I. Fernández-Domínguez, P. Tinnefeld, and G. P. Acuna, Plasmon-Assisted Förster Resonance Energy Transfer at the Single-Molecule Level in the Moderate Quenching Regime, Nanoscale 11, 7674 (2019).

[77] M. Sanz-Paz, J. Wenger, N. F. van Hulst, M. Mivelle, and M. F. Garcia-Parajo, Nanoscale Control of Single Molecule Förster Resonance Energy Transfer by a Scanning Photonic Nanoantenna, Nanophotonics 9, 4021 (2020).

[78] M. V. Rybin, K. L. Koshelev, Z. F. Sadrieva, K. B. Samusev, A. A. Bogdanov, M. F. Limonov, and Y. S. Kivshar, High- $Q$ Supercavity Modes in Subwavelength Dielectric Resonators, Phys. Rev. Lett. 119, 243901 (2017).

[79] B. Rolly, J.-M. Geffrin, R. Abdeddaim, B. Stout, and N. Bonod, Controllable Emission of a Dipolar Source Coupled with a Magneto-Dielectric Resonant Subwavelength Scatterer, Sci. Rep. 3, 3063 (2013).

[80] J. de Torres, M. Mivelle, S. B. Moparthi, H. Rigneault, N. F. Van Hulst, M. F. García-Parajó, E. Margeat, and J. Wenger, Plasmonic Nanoantennas Enable Forbidden Förster Dipole-Dipole Energy Transfer and Enhance the FRET Efficiency, Nano Lett. 16, 6222 (2016). 